\title{
Effects of dexmedetomidine hydrochloride on hemodynamics, postoperative analgesia and cognition in cesarean section
}

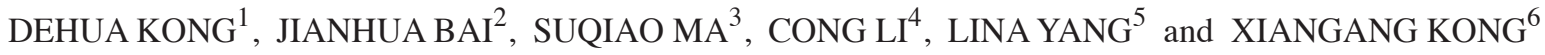 \\ ${ }^{1}$ Department of Obstetrics, Jining No. 1 People's Hospital, Jining, Shandong 272011; \\ Departments of ${ }^{2}$ Obstetrics, ${ }^{3}$ Oral and Maxillofacial Surgery, ${ }^{4}$ Otorhinolaryngology and ${ }^{5}$ Endocrinology, \\ People's Hospital of Zhangqiu District, Jinan, Shandong 250200; ${ }^{6}$ Department of Anesthesiology, \\ Jining No. 1 People's Hospital, Jining, Shandong 272011, P.R. China
}

Received January 11, 2018; Accepted June 22, 2018

DOI: $10.3892 / \mathrm{etm} .2018 .6363$

\begin{abstract}
The present study aims to investigate the effects of dexmedetomidine hydrochloride (Dex) on hemodynamics, postoperative analgesia and cognition in cesarean section. One hundred and two pregnant women who underwent cesarean section were selected from August 2016 to July 2017 in People's Hospital of Zhangqiu District and randomly divided into control group and observation group. Control group was anesthetized with bupivacaine hydrochloride, and morphine + ropivacaine hydrochloride were given postoperatively. Observation group received intraoperative anesthesia with bupivacaine hydrochloride and Dex, and Dex + ropivacaine hydrochloride were given for postoperative analgesia. Hemodynamic factors were compared between the two groups. Postoperative Ramsay sedation score, the incidence of adverse reactions and the incidence of transient neurological syndrome (TNS) were compared between the two groups. Montreal Cognitive Assessment (MoCA) and Mini-Mental State Examination (MMSE) scoring were performed to evaluate the cognitive function of the two groups. The mean arterial pressure (MAP) and visual analogue scale (VAS) scores of the observation group after anesthesia were significantly lower than those of control group $(\mathrm{P}<0.05)$. The Ramsay sedation score of the observation group was significantly better than that of control group at different time-points after surgery $(\mathrm{P}<0.05)$. Incidence of postoperative agitation in observation group was significantly lower than that in control group $(\mathrm{P}<0.05)$. Incidence of TNS in observation group was significantly lower than that in control group during 1 week after surgery $(\mathrm{P}<0.05)$. MoCA and MMSE scores of the observation group were better than that of control group at
\end{abstract}

Correspondence to: Dr Xiangang Kong, Department of Anesthesiology, Jining No. 1 People's Hospital, 6 Jiankang Road, Jining, Shandong 272011, P.R. China

E-mail: kdmqpa@163.com

Key words: dexmedetomidine hydrochloride, cesarean section surgery, hemodynamics, analgesia, cognitive function
1 day after operation $(\mathrm{P}<0.05)$. The use of Dex anesthesia in cesarean section can achieve more stable hemodynamic conditions during perioperative period and more obvious analgesic effect after operation. It also reduced the incidence of postoperative TNS and cognitive dysfunction, and had important clinical significance.

\section{Introduction}

Cesarean section refers to the surgical procedure of fetal removal by opening the lower abdominal cavity and uterus. This surgery is usually performed for high-risk pregnant women or those with abnormal delivery (1). Cesarean section operation is performed under anesthesia, and the main method is combined spinal-epidural anesthesia, which does not affect spontaneous breathing and cognition (2). Analgesic assistance is needed intraoperatively and postoperatively, and attentions should be paid on both analgesic effect and selection of drugs to protect the fetus (3). Intraoperative and postoperative pain should be carefully controlled after local anesthesia to relieve pain, achieve analgesic effect and facilitate the rapid recovery of pregnant women. However, postoperative complications, including transient neurological syndrome (TNS), agitation, delirium, anxiety and confusion, may happen $(4,5)$. In this study, dexmedetomidine hydrochloride (Dex) was used in cesarean section to investigate its effects on hemodynamic factors, postoperative analgesia and cognition.

\section{Materials and methods}

Clinical data. A total of 102 pregnant women who underwent cesarean section were selected from August 2016 to July 2017 in People's Hospital of Zhangqiu District (Jinan, China). Inclusion criteria: i) patients who underwent cesarean section; ii) single cephalic term pregnant women; and iii) patients who signed the written informed consent. Exclusion criteria: i) patients with serious drug allergy; ii) patients with obstetric complications and surgical complications; and iii) patients with severe mental illness. Patients were randomly divided into control group and observation group, with 51 cases in each group. There was no significant difference in general information between the two groups $(\mathrm{P}>0.05)$ (Table I). The 
Table I. Comparison of general information between the two groups.

\begin{tabular}{lccr}
\hline Items & Observation group $(\mathrm{n}=51)$ & Control group $(\mathrm{n}=51)$ & $\mathrm{t} / \chi^{2}$ test \\
\hline Age (years) & $20-38$ & $20-40$ & $\mathrm{P}$-value \\
Average age (years) & $28.46 \pm 5.63$ & $28.82 \pm 5.13$ & 0.338 \\
BMI $\left(\mathrm{kg} / \mathrm{m}^{2}\right)$ & $23.03 \pm 1.15$ & $23.16 \pm 1.29$ & 0.537 \\
Average gestational age (weeks) & $38.74 \pm 1.18$ & $38.49 \pm 1.23$ & 0.592 \\
Education (n, $\%)$ & & $6(11.76)$ & 0.298 \\
Junior high school and below & $8(15.69)$ & $23(45.10)$ & 0.517 \\
Secondary and high school & $20(39.21)$ & $22(43.14)$ & 0.772 \\
College or above & $23(45.10)$ & & \\
\hline
\end{tabular}

study was approved by the Ethics Committee of People's Hospital of Zhangqiu District (Jinan, China).

Methods. Both groups received combined spinal-epidural anesthesia. Patients were fixed in left lateral position. After conventional disinfection, L2-L3 intervertebral space was punctured. Epidural space was localized by using the resistance viscosity method. Arachnoid was punctured by using a spine needle. When cerebrospinal fluid reflux became smooth, $10 \mathrm{ml}$ bupivacaine hydrochloride (state approval no. H50020012; Southwest Pharmaceutical Co., Ltd., Chongqing, China) were injected into patients in each group. After that, $0.9 \%$ sodium chloride was injected into control group through a spine needle, while $4 \mu \mathrm{g}$ of Dex in the same volume of $0.9 \%$ sodium chloride was used in the observation group. Spine needle was removed after injection. Patients were adjusted to supine position and T6 plane. After the onset of anesthesia, cesarean section was performed to remove the baby from the mother.

Patients were given epidural continuous controlled analgesia after operation. In control group, morphine $(5 \mathrm{mg})+$ ropivacaine hydrochloride $(0.15 \%)$ (state approval no. H20050325; Guangdong Huarun Shunfeng Pharmaceutical Co., Ltd., Guangdong, China) in $100 \mathrm{ml} 0.9 \%$ sodium chloride solution was used. In control group, Dex $(1 \mu \mathrm{g} / \mathrm{kg})+$ ropivacaine hydrochloride $(0.15 \%)$ in $100 \mathrm{ml} 0.9 \%$ sodium chloride solution was used. A loading dose (2 mg morphine in control group and Dex at a dose of $0.1 \mu \mathrm{g} / \mathrm{kg}$ in observation group) $+\mathrm{a}$ background dose $(2 \mathrm{ml} / \mathrm{h})+$ PCA $(0.5 \mathrm{ml}$ each time with an interval of $15 \mathrm{~min}$ ) was performed for $48 \mathrm{~h}$.

\section{Evaluation method}

Heart rate (HR), mean arterial pressure (MAP), mean oxygen saturation $\left(\mathrm{SpO}_{2}\right)$, and visual analogue scale (VAS) scores were compared between the two groups before anesthesia (T1), 10 min after anesthesia (T2), when the baby was born (T3) and immediately after surgery (T4).

Ramsay sedation scoring was performed at 6, 12, 24 and 48 h after surgery: i) 1 point, irritability or anxiety; ii) 2 points, good directive force and cooperation ability; iii) 3 points, drowsiness, but can respond to instructions; iv) 4 points, can respond to strong sound stimuli or eyebrows reaction; v) 5 points, slow response to strong sound stimuli or eyebrows reaction; and vi) 6 points, no response to strong sound stimulation or light eyebrows (6).
Postoperative adverse reactions, including nausea, vomiting, hypoxemia, delayed recovery and postoperative agitation were observed in the two groups of patients. Postoperative TNS determination and grading: patients with dysfunction of the lower extremity on one or both sides; sensory disturbances and obvious pain, and the nature of pain is burning-like, press-like or radioactive pain. While electrophysiological examination results were normal. i) Grade I, single or bilateral dyskinesia and sensory disturbances were obvious, dyskinesia score $<26$ points, sensory disturbance score $<16$ points; ii) grade II, unilateral or bilateral motor impairment was not obvious, score $\geq 26$ points, but there was obvious sensory disturbance, sensory disturbance score $<16$ points; iii) grade III, unilateral or bilateral dyskinesia and sensory disturbances were not very obvious, only numbness and pain were observed (7).

Montreal Cognitive Assessment (MoCA) was used to evaluate the condition of patients at 1 day before and after operation through 8 aspects including executive ability, memory, attention, nomenclature, language, delayed recall, abstract thinking and directionality (8). Mini-Mental State Examination (MMSE) was used to evaluate the orientation force, language, memory, attention and computational power of patients (9). Both scores were negatively associated with the degree of cognitive impairment.

Statistical analysis. Data were processed by using SPSS 19.0 (IBM Corp., Armonk, NY, USA) software. Measurement data were expressed as mean \pm standard deviation (mean $\pm \mathrm{SD}$ ); t-test was used for the comparisons between groups. Count data were expressed as rate and processed by $\chi^{2}$ test. $\mathrm{P}<0.05$ was considered to be statistically significant.

\section{Results}

Comparison of hemodynamic factors between the two groups. There was no significant difference in $\mathrm{HR}$ and $\mathrm{SpO}_{2}$ between the two groups during perioperative period $(\mathrm{P}>0.05)$. The MAP score of the observation group after anesthesia was significantly lower than that of control group $(\mathrm{P}<0.05)$ (Tables II-IV).

Comparison of VAS scores during perioperative period. After anesthesia, patients in observation group showed significantly reduced pain score compared with those in control group $(\mathrm{P}<0.05)$ (Table V). 
Table II. Comparison of HR between the two groups at different time-points (mean $\pm \mathrm{SD}, \mathrm{bpm}$ ).

\begin{tabular}{lccccc}
\hline Groups & Cases & T1 & T2 & T3 & T4 \\
\hline Observation & 51 & $84.83 \pm 8.14$ & $74.53 \pm 7.48$ & $68.26 \pm 6.15$ & $74.86 \pm 5.52$ \\
Control & 51 & $85.46 \pm 8.15$ & $75.05 \pm 7.53$ & $67.87 \pm 6.27$ & $75.14 \pm 5.63$ \\
t-test & & 0.391 & 0.350 & 0.317 & 0.254 \\
P-value & & 0.697 & 0.727 & 0.752 & 0.800 \\
\hline
\end{tabular}

HR, heart rate.

Table III. Comparison of $\mathrm{SpO}_{2}$ between the two groups at different time-points (mean $\pm \mathrm{SD}, \%$ ).

\begin{tabular}{lccccc}
\hline Groups & Cases & T1 & T2 & T3 & T4 \\
\hline Observation & 51 & $97.47 \pm 0.44$ & $98.62 \pm 0.34$ & $97.42 \pm 0.38$ & $99.55 \pm 0.17$ \\
Control & 51 & $97.52 \pm 0.31$ & $98.57 \pm 0.26$ & $97.35 \pm 0.45$ & $99.58 \pm 0.28$ \\
t-test & & 0.663 & 0.834 & 0.849 & 0.654 \\
P-value & & 0.509 & 0.406 & 0.398 & 0.515 \\
\hline
\end{tabular}

$\mathrm{SpO}_{2}$, oxygen saturation.

Table IV. Comparison of MAP between the two groups at different time-points (mean $\pm \mathrm{SD}, \mathrm{mmHg}$ ).

\begin{tabular}{lccccc}
\hline Groups & Cases & T1 & T2 & T3 & T4 \\
\hline Observation & 51 & $66.67 \pm 3.52$ & $67.24 \pm 4.46$ & $65.27 \pm 3.28$ & $62.62 \pm 3.47$ \\
Control & 51 & $67.12 \pm 3.64$ & $79.67 \pm 4.63$ & $75.58 \pm 3.54$ & $69.35 \pm 3.35$ \\
t-test & & 0.635 & 13.808 & 15.257 & 2.562 \\
P-value & & 0.527 & $<0.001$ & $<.001$ & 0.012
\end{tabular}

MAP, mean arterial pressure.

Table V. Comparison of VAS score at different time-points (mean $\pm \mathrm{SD}$, points).

\begin{tabular}{|c|c|c|c|c|c|}
\hline Groups & Cases & $\mathrm{T} 1$ & $\mathrm{~T} 2$ & T3 & $\mathrm{T} 4$ \\
\hline Observation & 51 & $6.95 \pm 1.14$ & $0.36 \pm 0.13$ & $1.13 \pm 0.32$ & $2.13 \pm 1.12$ \\
\hline Control & 51 & $7.16 \pm 1.23$ & $0.73 \pm 0.25$ & $1.86 \pm 0.27$ & $3.56 \pm 1.17$ \\
\hline t-test & & 0.894 & 9.377 & 12.451 & 6.305 \\
\hline P-value & & 0.373 & $<0.001$ & $<0.001$ & $<0.001$ \\
\hline
\end{tabular}

VAS, visual analogue scale.

Comparison of analgesic conditions between the two groups. Ramsay sedation scores in observation group were significantly better than those in control group at different time-points $(\mathrm{P}<0.05)$ (Table VI).

Comparison of adverse reactions between the two groups. There was no significant difference in incidence of nausea and vomiting, hypoxemia and delayed recovery between the two groups $(\mathrm{P}>0.05)$. The incidence of postoperative agitation in observation group was significantly lower than that in control group $(\mathrm{P}<0.05)$ (Table VII).

Comparison of incidence of TNS between the two groups within 1 week after operation. The incidence of TNS was significantly lower in observation group than in control group $(\mathrm{P}<0.05)$ (Table VIII). 
Table VI. Comparison of postoperative Ramsay sedation scores between the two groups.

\begin{tabular}{lccccc}
\hline Groups & Cases & 6 h after operation & 12 h after operation & 24 h after operation & 48 h after operation \\
\hline Observation & 51 & $2.95 \pm 0.14$ & $4.76 \pm 0.23$ & $2.98 \pm 0.26$ & $2.63 \pm 0.22$ \\
Control & 51 & $2.16 \pm 0.23$ & $3.42 \pm 0.25$ & $2.65 \pm 0.26$ & $2.36 \pm 0.27$ \\
t-test & & 20.953 & 7.148 & 4.467 & 5.536 \\
P-value & $<0.001$ & $<0.001$ & $<0.001$ & $<0.001$ \\
\hline
\end{tabular}

Table VII. Comparison of adverse reactions between the two groups (n, \%).

\begin{tabular}{lccccc}
\hline Groups & Cases & Nausea and vomiting & Hypoxemia & Postoperative agitation & Delayed recovery \\
\hline Observation & 51 & $3(5.88)$ & $1(1.96)$ & $0(0.00)$ & $2(3.92)$ \\
Control & 51 & $5(9.80)$ & $3(5.88)$ & $6(11.76)$ & $4(7.84)$ \\
$\chi^{2}$ test & & 0.136 & 0.260 & 4.427 & 0.177 \\
P-value & 0.713 & 0.610 & 0.035 & 0.674 \\
\hline
\end{tabular}

Table VIII. Comparison of incidence of TNS between the two groups (n, \%).

\begin{tabular}{lccccc}
\hline Groups & Cases & I & II & III & Incidence of TNS \\
\hline Observation & 51 & $1(1.96)$ & $2(3.92)$ & $3(5.88)$ & $6(11.76)$ \\
Control & 51 & $4(7.84)$ & $5(9.80)$ & $7(13.73)$ & $16(31.37)$ \\
$\chi^{2}$ test & & & & 4.694 \\
P-value & & & & 0.030 \\
\hline
\end{tabular}

TNS, transient neurological syndrome.

Table IX. Comparison of MoCA and MMSE scores between the two groups (mean \pm SD, points).

\begin{tabular}{|c|c|c|c|c|c|c|c|c|}
\hline \multirow[b]{2}{*}{ Groups } & \multicolumn{4}{|c|}{$\mathrm{MoCA}$} & \multicolumn{4}{|c|}{ MMSE } \\
\hline & 1 day before & 1 day after & t-test & P-value & 1 day before & 1 day after & t-test & $\mathrm{P}$-value \\
\hline Observation & $28.14 \pm 1.23$ & $26.65 \pm 1.09$ & 6.475 & $<0.001$ & $30.12 \pm 1.12$ & $26.12 \pm 1.23$ & 17.172 & $<0.001$ \\
\hline Control & $28.13 \pm 1.14$ & $25.37 \pm 1.08$ & 12.552 & $<0.001$ & $29.78 \pm 1.13$ & $23.06 \pm 1.24$ & 28.606 & $<0.001$ \\
\hline t-test & 0.043 & 5.957 & & & 1.526 & 12.512 & & \\
\hline P-value & 0.966 & $<0.001$ & & & 0.130 & $<0.001$ & & \\
\hline
\end{tabular}

MoCA, Montreal Cognitive Assessment; MMSE, Mini-Mental State Examination.

Comparison of MoCA and MMSE scores between the two groups. Postoperative MoCA and MMSE scores of both groups were lower than those before surgery, and the decreases in control group were more significant than that in observation group $(\mathrm{P}<0.05)$ (Table IX).

\section{Discussion}

With the development of human society, the maternal delivery method has undergone significant changes. With the advantages of good muscle relaxation effects, exact analgesic effects and satisfactory postoperative analgesic effects, cesarean section has been more and more applied in clinical practices (10). Primiparas are usually affected by bad mood, resulting in longer labor course and uterine contractions and severe physical pain than those of multipara. Severe physical pain, anxiety, fear, tension and other adverse emotions can induce secretion of adrenal cortex hormones, endorphins, catecholamines and pain-causing substances, resulting in increased sensitivity to pain (11). Therefore, primiparas are usually treated with cesarean section to end delivery.

During perioperative period of cesarean section, surgical procedures can cause changes in heart rate and blood pressure (12). Dex is a highly selective $\alpha_{2}$-adrenergic receptor agonist that can suppress various surgical stress responses and maintain hemodynamic stability (13). Results of this study 
showed no significant difference in perioperative HR and $\mathrm{SpO}_{2}$ between the two groups $(\mathrm{P}>0.05)$. MAP and VAS scores in observation group after anesthesia were significantly lower than those in control group $(\mathrm{P}<0.05)$. This is because Dex can reduce the intraoperative hypertension reaction and activate the $\alpha_{2}$ receptor in the motor neuron complex on the dorsal medulla oblongata, so as to effectively reduce blood pressure. In addition, Dex can inhibit the release of adrenaline, so that the tension of the sympathetic nervous system will be reduced, resulting in analgesic and antiemetic effects.

Pain is severe with $48 \mathrm{~h}$ after cesarean section, and this pain can reduce uterine contractility and cause postpartum hemorrhage, leading to reduced uterine contractility, postpartum hemorrhage, reduced oxytocin secretion, reduced milk production and failure of breastfeeding (14). Without proper control, postoperative severe pain of cesarean section may lead to chronic pain. In Western countries, the incidence of chronic pain after caesarean section shows a continuous increasing trend (15). Therefore, proper postoperative analgesia is essential. Results of this study showed that the Ramsay sedation score of the observation group was significantly higher than that of the control group at 6,12,24 and $24 \mathrm{~h}$ after operation $(\mathrm{P}<0.05)$. This is because Dex can activate maternal $\mathrm{K}^{+}$channels and promote $\mathrm{K}^{+}$conduction, thereby activating an endogenous sleep-promoting pathway, resulting in a hypnotic effect. Dex can also enter cerebrospinal fluid to bind to $\alpha_{2}$ receptor to inhibit the release of histamine in the cortical and subcortical projection systems, thereby mediating conscious sedation. Dex can reduce sympathetic tone while enhancing vagal activity, thereby reducing excessive catecholamine and norepinephrine release, resulting in long-term analgesic effects (16).

Results of this study showed no significant difference in the incidence of adverse reactions such as nausea and vomiting, hypoxemia and delayed recovery between the two groups $(P>0.05)$. The incidence of postoperative agitation was significantly lower in observation group than in control group, and the incidence of TNS in observation group was significantly lower than that in control group $(\mathrm{P}<0.05)$. This is because Dex can inhibit $\mathrm{Ca}^{2+}$ influx in nerve endings, reduce excitability of the postsynaptic membrane, inhibit vasoconstriction and reduce temperature threshold of chills, resulting in anti-chills effects and inhibit the occurrence of postoperative agitation (17). Combined spinal-epidural anesthesia may cause damage to the nerve root, and narcotic drugs can easily deposit in spinal nerve root to block nerves. Intrathecal vulnerable area is posterior spinal nerve root, and nerve fibers have no myelination. Besides that, excessive air or saline may be injected to increase pressure within the spinal canal to cause damage to this area (18). Synergistic effects of Dex and local anesthetics can enhance the analgesic effect and reduce the use of local anesthetic, reduce the mild toxicity of narcotic drugs, thereby reducing the incidence of TNS.

Various discomforts during the postoperative recovery of cesarean section may cause sympathetic nervousness and central nervous system sensitization, resulting in various stress reactions. Activated inflammatory factors may cause inflammatory reactions. With the release of excessive inflammatory cytokines, they may migrate through the blood-brain barrier and cause inflammation in the hippocampus of the brain, resulting in cognitive dysfunction (19). Results of this study showed that postoperative MoCA and MMSE scores of both groups were lower than preoperative scores, and the decreases were more significant in control group than in observation group $(\mathrm{P}<0.05)$. This is because Dex has a certain anti-inflammatory effect, can inhibit the production of inflammatory cytokines, so as to reduce the damage to nervous system and protect brain. Dex is not harmful to central cholinergic neurons and fibers, and can improve the level of acetylcholine to participate in cognitive function, such as feeling, awakening, learning and judgment, so as to reduce cognitive impairment (20).

In conclusion, the use of Dex anesthesia in cesarean section can achieve stable hemodynamic conditions during perioperative period and obvious analgesic effect after operation. It also reduced the incidence of postoperative TNS and cognitive dysfunction.

\section{Acknowledgements}

Not applicable.

\section{Funding}

This study was supported by the Medical and Health Project of Jining Scientific and Technological Development Plan (2015-57-07).

\section{Availability of data and materials}

All data generated or analyzed during this study are included in this published article.

\section{Authors' contributions}

DK and JB designed the study. SM and CL collected the patients' data. LY and XK analyzed the patients' data. All authors read and approved the final manuscript.

\section{Ethics approval and consent to participate}

The present study was approved by the Ethics Committee of People's Hospital of Zhangqiu District (Jinan, China). Written informed consents were obtained from all patients.

\section{Patient consent for publication}

Not applicable.

\section{Competing interests}

The authors declare that they have no competing interests.

\section{References}

1. Betrán AP, Ye J, Moller AB, Zhang J, Gülmezoglu AM and Torloni MR: The increasing trend in caesarean section rates: Global, regional and national estimates: 1990-2014. PLoS One 11: e0148343, 2016.

2. Mueller NT, Whyatt R, Hoepner L, Oberfield S, Dominguez-Bello MG, Widen EM, Hassoun A, Perera F and Rundle A: Prenatal exposure to antibiotics, cesarean section and risk of childhood obesity. Int J Obes 39: 665-670, 2015. 
3. Scott JM: Regional versus general anaesthesia for caesarean section. Anaesthesia 49: 86, 1994.

4. Silbert BS, Evered LA and Scott DA: Incidence of postoperative cognitive dysfunction after general or spinal anaesthesia for extracorporeal shock wave lithotripsy. Br J Anaesth 113: 784-791, 2014.

5. Talakoub R, Golparvar M and Arshi R: The effect of early ambulation on the incidence of neurological complication after spinal anesthesia with lidocaine. J Res Med Sci 20: 383-386, 2015.

6. Sardesai SP, Patil KN and Sarkar A: Comparison of clonidine and dexmedetomidine as adjuncts to intravenous regional anaesthesia. Indian J Anaesth 59: 733-738, 2015.

7. Mao S, Zhu C and Chang Y: Effects of different anesthesia methods on postoperative transient neurological syndrome in patients with lumbar disc herniation. Exp Ther Med 14: 3112-3116, 2017.

8. Arevalo-Rodriguez I, Smailagic N, Roqué I Figuls M, Ciapponi A, Sanchez-Perez E, Giannakou A, Pedraza OL, Bonfill Cosp X and Cullum S: Mini-Mental State Examination (MMSE) for the detection of Alzheimer's disease and other dementias in people with mild cognitive impairment (MCI). Cochrane Database Syst Rev 3: CD010783, 2015.

9. Zhang J, Chong EKP and Tse DNC: Output MAI distributions of linear MMSE multiuser receivers in DS-CDMA systems. IEEE Trans Inf Theory 47: 1128-1144, 1998

10. Vogel JP, Betrán AP, Vindevoghel N, Souza JP, Torloni MR,Zhang J, Tunçalp Ö, Mori R, Morisaki N, Ortiz-Panozo E, et al; WHO Multi-Country Survey on Maternal and Newborn Health Research Network: Use of the Robson classification to assess caesarean section trends in 21 countries: A secondary analysis of two WHO multicountry surveys. Lancet Glob Health 3: e260-e270, 2015.

11. Hofmeyr GJ, Barrett JF and Crowther CA: Planned caesarean section for women with a twin pregnancy. Cochrane Database Syst Rev: Dec 19, 2015 (Epub ahead of print).

12. Bergholt T, Østberg B, Legarth J and Weber T: Danish obstetricians' personal preference and general attitude to elective cesarean section on maternal request: A nation-wide postal survey. Acta Obstet Gynecol Scand 83: 262-266, 2004.
13. Bell MT, Agoston VA, Freeman KA, Puskas F, Herson PS, Mares J, Fullerton DA and Reece TB: Interruption of spinal cord microglial signaling by alpha-2 agonist dexmedetomidine in a murine model of delayed paraplegia. J Vasc Surg 59: 1090-1097, 2014.

14. Niklasson B, Georgsson Öhman S, Segerdahl M and Blanck A: Risk factors for persistent pain and its influence on maternal wellbeing after cesarean section. Acta Obstet Gynecol Scand 94: 622-628, 2015

15. Moriyama K, Ohashi Y, Motoyasu A, Ando T, Moriyama K and Yorozu T: Intrathecal administration of morphine decreases persistent pain after cesarean section: A prospective observational study. PLoS One 11: e0155114, 2016.

16. Funai Y, Pickering AE, Uta D, Nishikawa K, Mori T, Asada A, Imoto $\mathrm{K}$ and Furue H: Systemic dexmedetomidine augments inhibitory synaptic transmission in the superficial dorsal horn through activation of descending noradrenergic control: An in vivo patch-clamp analysis of analgesic mechanisms. Pain 155 : 617-628, 2014.

17. Sun L, Guo R and Sun L: Dexmedetomidine for preventing sevoflurane-related emergence agitation in children: A meta-analysis of randomized controlled trials. Acta Anaesthesiol Scand 58: 642-650, 2014.

18. Goeller JK, Joselyn A, Martin DP, Bhalla T, Dairo O, Herz DB, Alpert SA and Tobias JD: Epidural pressure changes following caudal blockade: A prospective, observational study. J Anesth 30: 578-582, 2016.

19. Chu MH, Tsang S, Wu WK and Chan M: Anaesthetic implications of mild cognitive impairment. Anaesth Intensive Care 44: 539-541, 2016.

20. Kimura M, Saito S and Obata H: Dexmedetomidine decreases hyperalgesia in neuropathic pain by increasing acetylcholine in the spinal cord. Neurosci Lett 529: 70-74, 2012.

This work is licensed under a Creative Commons Attribution-NonCommercial-NoDerivatives 4.0 International (CC BY-NC-ND 4.0) License. 